\title{
Ready-Simulation Is Not Ready to Express a Modular Refinement Relation
}

\author{
Françoise Bellegarde, Jacques Julliand, and Olga Kouchnarenko \\ LIFC, Univ. Franche-Comté, 16, route de Gray, 25030 Besançon Cedex France \\ \{bellegar, julliand, kouchna\}@lifc.univ-fcomte.fr, \\ http://lifc.univ-fcomte.fr
}

\begin{abstract}
The B method has been successfully used to specify many industrial applications by refinement. Previously, we proposed enriching the B event systems by formulating its dynamic properties in $L T L$. This enables us to combine model-checking with theorem-proving verification technologies. The model-checking of $L T L$ formulae necessitates that the $\mathrm{B}$ event system semantics is a transition system. In this paper, we express the refinement relation by a relationship between transition systems. A result of our study shows that this relation is a special kind of simulation allowing us to exploit the partition of the reachable state space for a modular verification of $L T L$ formulae. The results of the paper allow us to build a bridge between the above view of the refinement and the notions of observability characterized as simulation relations by Milner, van Glabbeek, Bloom and others. The refinement relation we define in the paper is a ready-simulation generalization which is similar to the refusal simulation of Ulidowsky. The way the relation is defined allows us to obtain a compositionality result w.r.t. parallel composition operation.
\end{abstract}

For complex systems, it is important in practice to associate a design by refinement with a design by a parallel composition of their components. This refinement relation has two main applications:

- it allows the splitting of the refined transition system into modules;

- it allows the construction of complex systems by a parallel composition of components.

It makes sense to qualify the refinement relation as being modular.

\section{Introduction}

In this paper, we express the refinement semantics as a relation between transition systems because we want to associate the verification of $L T L$ formulae in the framework of a refinement design of reactive systems.

The B refinement method has been successfully used to specify many reactive systems: case studies such as an elevator [2], an industrial automatism [3], a steam-boiler case study [4, as well as industrial applications such as MÉTÉOR 7 ] by Matra Transport International, and the SPECTRUM project [23] by GECMarconi Avionics Limited. 
The B refinement method [1, 6] is used to specify reactive systems by event systems. In [12, 14, we propose to enrich this specification wiht dynamic properties formulated in the Linear Temporal Logic $(L T L)$. We can then combine model-checking with theorem proving techniques. For that the verification has to take place both at the syntactic level for theorem proving and at the operational level for the verification of $L T L$ formulae by model-checking. At the operational level, the specification is expressed with a transition system. At this level, the refinement relates transition systems between themselves. In 14, 18, we show that the refinement splits the refined transition system into modules. This allows us to verify some $L T L$ properties separately on each module and to let what remains to be proved. For that, the refinement needs to be defined between transition systems. Therefore, the refinement verification takes place also at the operational level.

The results of the paper allow us to build a bridge between the above view of the refinement and the notions of observability characterized as simulation relations by Milner, van Glabbeek, Bloom and others.

We define the refinement relation as a simulation which allows us to exploit a partition of the refined reachable state space. With such a partition we are able to avoid the model-checking blow-up by verifying $L T L$ formulae in a modular way [14]. Moreover, we want this refinement relation to be compositional w.r.t. parallel composition through refinement. That is why we call "modular" our refinement relation.

This paper is organized as follows. After giving preliminary notions in Section 2 Section 3 defines the behavior semantics of the transition systems derived from the B refinement design. Then, we define the transition system modular refinement relation in Section 4 Its expressiveness is studied in Sections 5 and 6 . In Section 7 we illustrate the use of our framework on the example of the robot carrying parts from an arrival device towards two exit devices. Then, Section 8 explains how the refinement relation is used in the context of a verification tool set which combines automatic-proof and model-checking technologies. We end by some related works and some perspectives.

\section{Preliminaries}

In this paper we are concerned with a relationship between transitions systems which is a binary relation on their sets of states. In this framework, a predicate transformer is a function transforming sets of states into sets of states.

A transition systems is a pair $\langle S, \rightarrow\rangle$, where $S$ is a set of states and $\rightarrow$ is a transition relation on $S(\rightarrow \subseteq S \times S)$.

Definition 1. (pre $[\psi]$ and post $[\psi]$ predicate transformers) Given a relation $\psi$ between two set of states $S_{1}$ and $S_{2}\left(\psi: S_{1} \times S_{2}\right)$, we define pre $[\psi]: 2^{S_{2}} \times 2^{S_{1}}$ and post $[\psi]: 2^{S_{1}} \times 2^{S_{2}}$ by 


$$
\begin{aligned}
& \text { - pre }[\psi] \stackrel{\text { def }}{=} \lambda X .\left\{q_{1} \in S_{1} \text { s.t. }\left(\exists q_{2} \in X \text { s.t. } q_{1} \psi q_{2}\right)\right\} \\
& \text { - post }[\psi] \stackrel{\text { def }}{=} \lambda X .\left\{q_{2} \in S_{2} \text { s.t. }\left(\exists q_{1} \in X \text { s.t. } q_{1} \psi q_{2}\right)\right\}
\end{aligned}
$$

So, for $S_{2}^{\prime} \subseteq S_{2}$, pre $[\psi]\left(S_{2}^{\prime}\right)$ represents the set of predecessors of the states of $S_{2}^{\prime}$ via the relation $\psi$, and, for $S_{1}^{\prime} \subseteq S_{1}, \operatorname{post}[\psi]\left(S_{1}^{\prime}\right)$ represents the set of successors of the states of $S_{1}^{\prime}$ via $\psi$. Some useful results concerning the pre and post predicate transformers can be found in 22 as, for example, the two following propositions.

Proposition 1. For any relation $\psi$ from a set $S_{1}$ to a set $S_{2}\left(\psi \subseteq S_{1} \times S_{2}\right)$, we have:

- For any $X_{1}, X_{2}$ subsets of $S_{2}$, pre $[\psi]\left(X_{1} \cup X_{2}\right)=\operatorname{pre}[\psi]\left(X_{1}\right) \cup \operatorname{pre}[\psi]\left(X_{2}\right)$.

- For any $X_{1}, X_{2}$ subsets of $S_{1}$, post $[\psi]\left(X_{1} \cup X_{2}\right)=\operatorname{post}[\psi]\left(X_{1}\right) \cup \operatorname{post}[\psi]\left(X_{2}\right)$.

We adopt the following notations:

- We denote by $I d_{S}$ the identity function on $2^{S}$.

- We denote by $\widetilde{\alpha}$ the dual of a function $\alpha: 2^{S_{1}} \rightarrow 2^{S_{2}}$ that is $\widetilde{\alpha} \stackrel{\text { def }}{=} \lambda X . \overline{\alpha(\bar{X})}$.

- We denote the composition of two relations $\psi \subseteq S_{1} \times S_{2}$ and $\phi \subseteq S_{2} \times S_{3}$ by their juxtaposition $\psi \phi$.

- We denote the composition of two predicate transformers $\alpha: X \rightarrow Y$ and $\beta: Y \rightarrow Z$ by $\beta \circ \alpha: X \rightarrow Z$.

Proposition 2. Let be $\rightarrow \subseteq S \times S$. Then pre $\left[\rightarrow^{2}\right]=\operatorname{pre}[\rightarrow] \circ \operatorname{pre}[\rightarrow]$.

We give hereafter the definition of Galois connections and some results about them. More information can, e.g., be found in [19]21].

Definition 2. (Galois connections) Let $S_{1}$ and $S_{2}$ be two sets of states. A connection from $2^{S_{1}}$ to $2^{S_{2}}$ is a pair of monotonic functions $(\alpha, \gamma)$, where $\alpha$ : $2^{S_{1}} \rightarrow 2^{S_{2}}$ and $\gamma: 2^{S_{2}} \rightarrow 2^{S_{1}}$, such that $I d_{S_{1}} \subseteq \gamma \circ \alpha$ and $\alpha \circ \gamma \subseteq I d_{S_{2}}$.

It is well-known that $\alpha$ and $\gamma$ determine each other in a unique manner. These characterizations allow obtaining in [16] a proposition showing the links between the binary relation $\psi$ from $S_{2}$ to $S_{1}$ and the connections from $2^{S_{2}}$ to $2^{S_{1}}$ in term of predicate transformers pre and post.

Proposition 3. (Connections generated by a binary relation on states) If $\psi \subseteq S_{1} \times S_{2}$, then the pair (post $[\psi]$, $\left.\widetilde{\text { pre }}[\psi]\right)$ is a connection from $2^{S_{1}}$ to $2^{S_{2}}$, and $($ pre $[\psi]$, post $[\psi])$ is a connection from $2^{S_{2}}$ to $2^{S_{1}}$.

\section{Behavioral Semantics of Systems Derived from the B Design}

In this paper we look at the B refinement at the operational semantic level since it splits the refined transition system into modules. This allows a modular verification of some $L T L$ properties. 
We define an operational semantics of a B specification under the form of a labeled transition system. We denote by $S$ a set of states. We introduce a finite set of variables $V \stackrel{\text { def }}{=}\left\{x_{1}, \ldots, x_{n}\right\}$. Let $l$ be an injective function which allows us to give values to the states as a conjunction of variable/value equalities. Let $q$ be a state, and $v_{1}, \ldots, v_{n}$ be values, then $l(q)(l(q)$ holds in $q)$ is defined by $x_{1}=v_{1} \wedge \cdots \wedge x_{n}=v_{n}$. Usually, to describe $L T L$ formulae semantics, the set of all the propositions holding in a state $q$ is considered to be a label of $q$. We consider one of them $l(q)$ since, then, we know that a proposition $P$ holds in $q$ by $l(q) \Rightarrow P$ holds.

We call invariant a predicate $I$ which holds on each state, and, as such, formulates a safety property of the system. A specification in B always requires such an invariant. A predicate $I$ is an invariant of an interpreted transition system iff $\forall q \in S, l(q) \Rightarrow I$.

Let $A c t \stackrel{\text { def }}{=}\{a, b, \ldots\}$ be a nonempty alphabet of interpreted actions. The actions affect state variables. A labeled transition relation $\rightarrow(\subseteq S \times$ Act $\times$ $S$ ) is defined as a set of triples $\left(q, a, q^{\prime}\right)$ (written " $q \stackrel{a}{\rightarrow} q^{\prime \prime}$ "). The interpreted transition system $T S=\langle S, A c t, \rightarrow, l\rangle$ has the state space $S \stackrel{\text { def }}{=}\left\{q, q^{\prime}, q_{1}, \ldots\right\}$, labeled transition relation $\rightarrow \subseteq S \times A c t \times S$, and the state interpretation function $l$.

The transition relation $\rightarrow$ can be extended on a sequence of transitions in the standard way: $q^{\prime}$ is reachable from $q$, written $q^{\prime} \in(\rightarrow)^{*}(q)$, (or there is a path $\sigma$ from $q$ to $q^{\prime}$ ) if there exist states $q_{1}, \ldots, q_{n}$ and transitions $t_{1}, \ldots, t_{n-1}$ respectively labeled by $a_{1}, \ldots, a_{n-1}$ such that

$$
(q=) q_{1} \stackrel{a_{1}}{\rightarrow} q_{2} \ldots \stackrel{a_{n-1}}{\rightarrow} q_{n}\left(=q^{\prime}\right) .
$$

We note $\Upsilon_{T S}(q)$ the set of the paths of the transition system TS beginning in $q$. Given the path $\sigma=q \stackrel{a}{\rightarrow} q^{\prime} \stackrel{b}{\rightarrow} q^{\prime \prime} \stackrel{c}{\rightarrow} \ldots$ in $\Upsilon_{T S}(q)$, we define its trace, note $\operatorname{tr}(\sigma)$ by $\operatorname{tr}(\sigma) \stackrel{\text { def }}{=} a b c \ldots$.

Moreover, given a state $q$, any proposition $P$ such that $l(q) \Rightarrow P$ can be used to verify a $L T L$ formula along a path which contains $q$ in its trace.

As usual, the inverse relation $(\rightarrow)^{-1}$ denotes the predecessor relation on states, and we can say that $q^{\prime}$ is reachable from $q, q(\rightarrow)^{*} q^{\prime}$, iff $q^{\prime}\left((\rightarrow)^{*}\right)^{-1} q^{\prime}$.

\section{Modular Refinement Relation}

In this section we consider two interpreted transition systems $T S_{1}=\left\langle S_{1}, A c t_{1}, \rightarrow_{1}\right.$ ,$\left.l_{1}\right\rangle$ and $T S_{2}=\left\langle S_{2}, A c t_{2}, \rightarrow_{2}, l_{2}\right\rangle$ giving the operational semantics of two systems at two levels of refinement. We say that $T S_{2}$ is a refinement of $T S_{1}$.

The syntactical requirements of the $\mathrm{B}$ refinement are expressed as follows:

1. The refinement introduces new actions, so $A c t_{1} \subseteq A c t_{2}$.

2. The invariant $I_{2}$, commonly called the gluing invariant (for instance, cf. [6]), expresses how the variables from the two interpreted transition systems are linked. More precisely, the invariant of the refined system is $I_{2} \wedge I_{1}$. 


\subsection{Modular Refinement as State Space Partition}

We exploit the B refinement so that we can build a partition of the state space.

First, we define a binary relation $\mu \subseteq S_{2} \times S_{1}$ allowing us to express the relation between values of two consecutive interpreted transition systems.

Definition 3. (Glued states) The state $q_{2} \in S_{2}$ is glued to $q_{1} \in S_{1}$, written as $q_{2} \mu q_{1}$, iff $l\left(q_{2}\right) \wedge I_{2} \Rightarrow l\left(q_{1}\right)$.

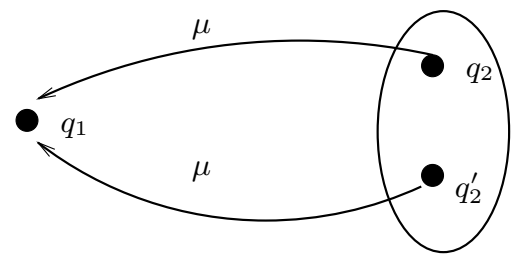

Fig. 1. State space partition idea

The glue relation $\mu$ allows us to define an equivalence relation $\sim_{\mu}$ between states of the second level transition system. Formally, two states $q_{2}$ and $q_{2}^{\prime}$ of the $T S_{2}$ are equivalent iff there exists a state $q_{1}$ of $T S_{1}$ s.t. $q_{2} \quad \mu \quad q_{1}$ and $q_{2}^{\prime} \quad \mu q_{1}$ (see Figure 11). Indeed, we get an equivalence since giving two distinct states $q_{2}$ and $q_{2}^{\prime}$ we cannot have two distinct states $q_{1}$ and $q_{1}^{\prime}$ satisfying the above implication. When there are states of $T S_{2}$ glued to the state $q_{1}$ of $T S_{1}$, the state $q_{1}$ gives its name $q_{1}$ to an element of the partition.

Definition 4. (Equivalence class name) Let $X$ be an equivalence class of $S_{2} / \sim_{\mu}$. The state $q_{1} \in S_{1}$ is the name of $X$ iff, for a state $q_{2} \in X$, we have $q_{2} \mu q_{1}$.

\subsection{Modular Refinement as a Relation}

In this section we consider the refinement of an interpreted transition system as a simulation and we motivate it as a path set inclusion. Our purpose is to define a refinement relation to be a simulation allowing us to exploit the partition of the reachable state space for a modular verification of $L T L$ formulae. For that, we restrict $\mu$ into a relation $\rho$ which relates a refined transition system to one of its abstraction, and, we restrict $\rho^{-1}$ into a relation $\xi$ which relates a transition system to one of its refinement. These relations are useful to distinguish some elements of the partition of the state space.

This partition is used first, to prove an invariant of a module, and second, to verify propositional components of a $L T L$ formula which is verified on a module. 
From a Refined Transition System to One of Its Abstraction Let us call $\rho$ a relation included into $\mu$ between the states of $T S_{2}$ and $T S_{1}$ which satisfies the following requirements:

1. In order to describe the refinement, we keep the transitions of $T S_{2}$ labeled over $A c t_{1}$ but the new ones (from $A c t_{2} \backslash A c t_{1}$ ) introduced by the refinement are considered as non observable $\tau$ moves. These $\tau$ moves hide the transitions of the modules viewed as interpreted transition systems. Indeed, the transitions of a module with state space $S$ are the $\tau$ moves between the states of $S$ (cf. Figure 2).
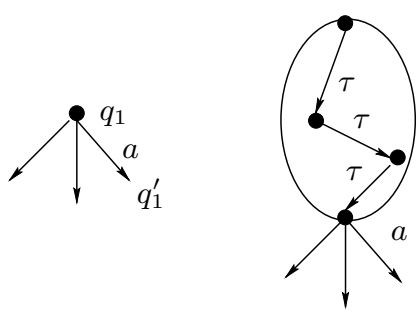

Fig. 2. Silent moves

2. In the above modules, it is certainly not desirable that $\tau$ moves take control forever. So, we want no deadlock and no infinite path of $\tau$ moves.

Let $A c t_{1 \tau} \stackrel{\text { def }}{=} A c t_{1} \cup\{\tau\}$. For each $a \in A c t_{1 \tau}$, we note $q \stackrel{a}{\Longrightarrow} q^{\prime}$ when there is $n \geq 0$ such that $q \stackrel{\tau^{n} a}{\longrightarrow} q^{\prime}$. Intuitively, a transition $\stackrel{a}{\Longrightarrow}$ allows us to absorb a finite number of $\tau$ before the action $a$. Notice that Milner in [17] uses $\stackrel{a}{\Longrightarrow}$ for $\stackrel{\tau^{n} a \tau^{m}}{\longrightarrow}$ with $n, m \geq 0$ but we force $m$ to be equal to 0 . The reason is that the occurrence of an action $a$ determines the end of a path of a module. Notice that all the paths of a module are finite ones.

Definition 5. Let $T S_{1}=\left\langle S_{1}, A c t_{1}, \rightarrow_{1}, l_{1}\right\rangle$ and $T S_{2}=\left\langle S_{2}, A c t_{1 \tau}, \rightarrow_{2}, l_{2}\right\rangle$ be

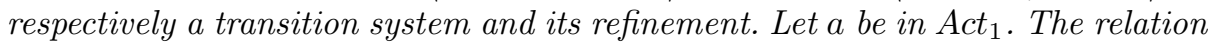
$\rho \subseteq S_{2} \times S_{1}$ is defined as the greatest binary relation included into $\mu$ and satisfying the following clauses:

1. (strict transition refinement) $\left(q_{2} \rho q_{1} \wedge q_{2} \stackrel{a}{\rightarrow}{ }_{2} q_{2}^{\prime}\right) \Rightarrow\left(\exists q_{1}^{\prime}\right.$ s.t. $q_{1} \stackrel{a}{\rightarrow} 1$ $\left.q_{1}^{\prime} \wedge q_{2}^{\prime} \rho q_{1}^{\prime}\right)$

2. (stuttering transition refinement)

$\left(q_{2} \rho q_{1} \wedge q_{2} \stackrel{\tau}{\rightarrow}_{2} q_{2}^{\prime} \stackrel{a}{\Longrightarrow} 2 q_{2}^{\prime \prime}\right) \Rightarrow\left(\exists q_{1}^{\prime}\right.$ s.t. $\left.q_{1} \stackrel{a}{\rightarrow}_{1} q_{1}^{\prime} \wedge q_{2}^{\prime} \rho q_{1} \wedge q_{2}^{\prime \prime} \rho q_{1}^{\prime}\right)$ 


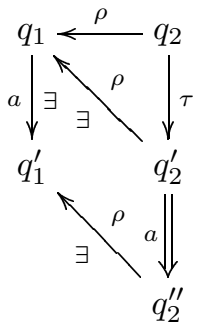

3. (lack of new deadlock) $\left(q_{2} \rho q_{1} \wedge q_{2} \nrightarrow_{2}\right) \Rightarrow\left(q_{1} \nrightarrow_{1}\right)$

4. (non $\tau$-divergence) $q_{2} \rho q_{1} \Rightarrow \neg\left(q_{2} \stackrel{\tau}{\rightarrow} q_{2}^{\prime} \stackrel{\tau}{\rightarrow} q_{2}^{\prime \prime} \stackrel{\tau}{\rightarrow} \cdots \stackrel{\tau}{\rightarrow} \cdots\right)$

Notice that the presence of Clause 4 guarantees the monotonicity of an iterative construction of the relation $\rho$ and, this way, the existence of this relation.

The relation $\rho$ implements the modular refinement viewed as a path set inclusion. Given $\Upsilon_{T S_{1}}$ and $\Upsilon_{T S_{2}}$, sets of paths of $T S_{1}$ and $T S_{2}$, we can see that

1. Clauses 1 and 2 of $\rho$-definition mean that every path of $\Upsilon_{T S_{2}}$ refines some path in $\Upsilon_{T S_{1}}$ (see Figure 3 ).

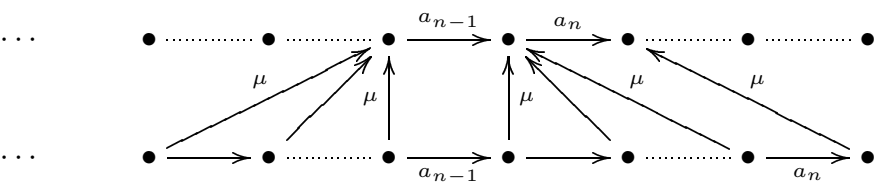

Fig. 3. Path refinement.

2. Clause 4 means that the refinement does not authorize infinite new paths composed only with new transitions in $T S_{2}$. In terms of graph, such infinite paths are cycles, so, there always must be a way out of these cycles, if any, by a strongly fair transition since such a transition, which is always eventually taken, forbids the infinite loop.

3. Clause 3 implies that any deadlock in $\Upsilon_{T S_{2}}$ corresponds to a deadlock in $\Upsilon_{T S_{1}}$ which means that new deadlocks are forbidden.

\section{From an Abstract Transition System to One of Its Refinement}

Definition 6. Let $T S_{1}=\left\langle S_{1}, A c t_{1}, \rightarrow_{1}, l_{1}\right\rangle$ and $T S_{2}=\left\langle S_{2}\right.$, Act $\left._{1 \tau}, \rightarrow_{2}, l_{2}\right\rangle$ be respectively a transition system and its refinement. Let a be in Act . The relation $_{1}$ $\xi \subseteq S_{1} \times S_{2}$ is the greatest binary relation included into $\rho^{-1}$ and satisfying the following clause: 
(non-determinism) $\left.\left(q_{1} \stackrel{a}{\rightarrow} 1 q_{1}^{\prime} \wedge q_{1} \xi q_{2}\right)\right) \Rightarrow\left(\exists q_{2}^{\prime}, q_{2}^{\prime \prime} \quad\right.$ s.t. $q_{1} \xi q_{2}^{\prime} \wedge q_{2}^{\prime} \stackrel{a}{\Longrightarrow} 2$ $\left.q_{2}^{\prime \prime} \wedge q_{1}^{\prime} \xi q_{2}^{\prime \prime}\right)$

The construction stays monotonic.

Again, we explain the $\xi$ relation in terms of the path set inclusion. If there is an internal non-deterministic choice among the transitions of $\Upsilon_{T S_{1}}$ which begins at the same state $q_{1}$ (see Definition 6), then there exists (at least) one of these transitions which is refined by some path $\sigma_{2} \in \Upsilon_{T S_{2}}$ (see Figure 4).

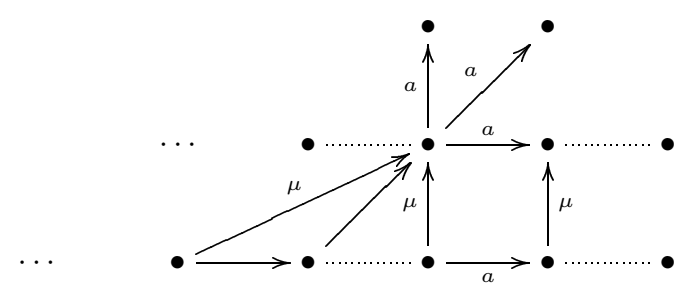

Fig. 4. Internal non-deterministic choice refinement.

Intuitively, the above notions of refinement mean that we observe the system more often taking into account additional details about its behavior. In terms of traces, the trace of the path $\sigma_{1}$ is embedded into the trace of the transitions in $\sigma_{2}$, or, in other words, we find the path $\sigma_{1}$ by removing the transitions labeled by new (from $A c t_{2} \backslash A c t_{1}$ ) actions from the path $\sigma_{2}$ but this holds only if the transition systems are deterministic. In the presence of a non-deterministic choice, it is possible that some traces disappear among a non-deterministic set of transitions, but at least one trace remains.

The relation $\xi$ allows us to define some elements of a partition of the refined state space $S_{2} / \sim_{\mu}$ as modules, i.e., $X \in S_{2} / \sim_{\mu}$ of name $q_{1} \in S_{1}$ (i.e. the equivalence class name, see Definition (4) is also a module (named $q_{1}$ ) if and only if, for all the states $q_{2}$ in $X$, we have $q_{1} \xi q_{2}$. So, from a designer point of view, " $\xi$ holds" means that $T S_{1}$ is refined by $T S_{2}$.

As a consequence of the definition of $\xi$, there can be no deadlock and no livelock inside a module. Moreover, each path $\tau^{*} a$ refines a $a$-transition beginning in $q_{1}$. Finally, the set of the labels of all the transitions beginning in $q_{1}$ is equal to the set of the labels $a$ of all the paths $\tau^{*} a$. This last consequence will be shown in Section 5.2 . 


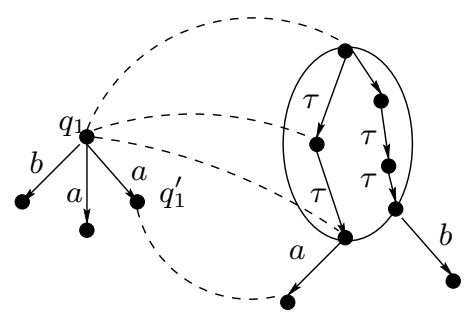

Fig. 5. Module

\section{Modular Refinement as a Simulation}

In this section, we consider the modular refinement relation $\eta \stackrel{\text { def }}{=} \xi^{-1}$. So, from a designer point of view, $\eta$ holds means that $T S_{2}$ refines $T S_{1}$. We show that the modular refinement relation can be viewed as a special kind of $\tau$-simulation which is derived from the observational equivalence of Milner [17] between a refined transition system and its abstraction. Finally, it is a generalization of the ready simulation of [10].

\subsection{Modular Refinement as a $\tau$-Simulation}

In this section, we are interested in a view of the modular refinement as a $\tau$ simulation from $T S_{2}$ to $T S_{1}$.

From the relation $\eta$, Proposition 3 allows us to generate a Galois connection $($ post $[\eta], \widetilde{\text { pre }}[\eta])$. The construction is inspired by [16]. Notice that the above authors are looking for building an abstraction when we are interested in a modular refinement. We choose this approach to show a $\tau$-simulation because we see better the role of the modules by the way of the predicate transformers post $[\eta]$ and $\widetilde{\text { pre }}[\eta]$. Notice that the Milner's approach observes each time two related states, so it does not allow to consider a whole module (a set of states) which is related to one abstract state.

Let $X$ be a subset of the refined state space $S_{2}\left(X \subseteq S_{2}\right)$. Then post $[\eta](X)$ is the set of the module names, i.e.,

$$
\operatorname{post}[\eta] \stackrel{\text { def }}{=} \lambda X .\left\{q_{1} \in S_{1} \text { s.t. }\left(\exists q_{2} \in X \text { s.t. } q_{2} \eta q_{1}\right)\right\}
$$

Let $X$ be a subset of the abstract state space $S_{1}\left(X \subseteq S_{1}\right)$. Then $\widetilde{p r e}[\eta](X)$ is the union of the state space of the modules named in $X$, i.e.,

$$
\begin{aligned}
\widetilde{p r e}[\eta] \stackrel{\text { def }}{=} \lambda X . \bigcup_{i \in I} X_{i} \text { s.t. } & \\
& \left(\forall i . i \in I \Rightarrow X_{i} \in S_{2} / \sim_{\mu}\right) \wedge\left(\forall q_{2} . q_{2} \in X_{i} \Rightarrow\left(\exists q_{1} \in X \text { s.t. } q_{2} \eta q_{1}\right)\right)
\end{aligned}
$$

By using this Galois connection we show that the modular refinement is a kind of $\tau$-simulation which is derived from the observational equivalence of Milner [17]. Now we give the definition of a $\eta$-simulation. 


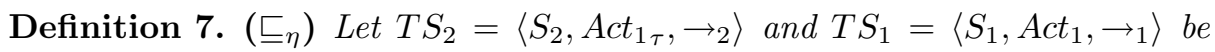
two transition systems and $\eta$ be a relation from $S_{2}$ to $S_{1}\left(\eta \subseteq S_{2} \times S_{1}\right)$. Define $S_{2} \sqsubseteq_{\eta} S_{1}$ if and only if $\left((\stackrel{\tau}{\rightarrow} 2)^{*}\right)^{-1}\left(\stackrel{a}{\rightarrow}_{2}\right)^{-1} \eta \subseteq \eta\left(\stackrel{a}{\rightarrow}_{1}\right)^{-1}$ for all $a \in$ Act $_{1}$.

If $S_{2} \sqsubseteq_{\eta} S_{1}$, we say that $S_{2} \eta$-simulates $S_{1}$. This $\eta$-simulation is closely related to the $\tau$-simulation in the sense of Milner. The difference is that in the tausimulation the action $a$ can be the empty action, and that $\tau$-transitions can follow $a$.

In Theorem 1, we show that, by modular refinement, $S_{2} \eta$-simulates $S_{1}$. For that, we need another predicate transformer in the refined transition system. Recall that a refined transition system $T S_{2}$ has silent moves whereas an abstract transition system $T S_{1}$ has no silent moves. For $T S_{2}$, the predicate transformer pre $\left[\rightarrow_{2}\right]$ is extended to pre $^{\tau^{*} a}\left[\rightarrow_{2}\right]$ to take into account the silent moves.

Definition 8. (pre $\tau^{\tau^{*} a}\left[\rightarrow_{2}\right]$ predicate transformer) Given the relation $\rightarrow_{2}$ : $S_{2} \times S_{2}$, we define pre $\tau^{\tau^{*}}\left[\rightarrow_{2}\right]: S_{2} \times S_{2}$ by pre $^{\tau^{*} a}\left[\rightarrow_{2}\right] \stackrel{\text { def }}{=} \lambda X_{2} \cdot\left\{q_{2} \in S_{2}\right.$ s.t. $\left(\exists q_{2}^{\prime} \in X_{2}\right.$ s.t. $\left.\left.q_{2}^{\prime} \stackrel{a}{\Longrightarrow} q_{2}\right)\right\}$.

The definition of $\operatorname{pre}^{\tau^{*} a}$ is not a problem because there is no infinite $\tau$-path (see Clause 4 of Definition 51. Notice that Proposition 1 applies to pre $^{\tau^{*} a}\left[\rightarrow_{2}\right]$ by finite composition of pre $\left[\rightarrow_{2}\right]$ because of Proposition 2,

We need the following lemma to prove that $S_{2} \eta$-simulates $S_{1}$.

Lemma 1. Let $\eta$ be the relation between the two transition systems $T S_{2}=$ $\left\langle S_{2}\right.$, Act $\left._{1 \tau}, \rightarrow_{2}, l_{2}\right\rangle$ and $T S_{1}=\left\langle S_{1}\right.$, Act $\left._{1}, \rightarrow_{1}, l_{1}\right\rangle$. Let $(\alpha, \gamma)$ be the Galois connection (post $[\eta], \widetilde{\text { pre }}[\eta])$. Then, we have

$$
\alpha \circ \operatorname{pre}^{\tau^{*} a}\left[\rightarrow_{2}\right] \circ \gamma \subseteq \operatorname{pre}\left[\rightarrow_{1}\right]
$$

Proof. Let $q_{i}$ be the name of a module $Y_{i}$ (see Definition 4). Then

$$
\alpha \circ \operatorname{pre}^{\tau^{*} a}\left[\rightarrow_{2}\right]\left(Y_{i}\right)
$$

gives the modules names of the set $P=\operatorname{pre}^{\tau^{*} a}\left[\rightarrow_{2}\right]\left(Y_{i}\right)$ by Definition of $\alpha$. All the elements of the set $P$ are related to a predecessor of $q_{i}$ by Clauses 11 and 2 of Definition 5. Therefore, this is included into the set pre $\left[\rightarrow_{1}\right]\left(\left\{q_{i}\right\}\right)$.

Let $X$ be a subset of the abstract state space $S_{1}\left(X \subseteq S_{1}\right)$. Then, $\gamma(X)=$ $\bigcup_{i \in I} Y_{i}$ where the $Y_{i}$ 's are the modules in $S_{2}$ the names of which are elements $\left\{q_{1}, q_{2}, \ldots, q_{i}, \ldots\right\}$ of $X$. Therefore,

$$
\alpha \circ \operatorname{pre}^{\tau^{*} a}\left[\rightarrow_{2}\right] \circ \gamma(X)=\alpha \circ \operatorname{pre}^{\tau^{*} a}\left[\rightarrow_{2}\right]\left(\bigcup_{i \in I} Y_{i}\right)
$$

which is equal to $\bigcup_{i \in I}\left(\alpha \circ \operatorname{pre}^{\tau^{*} a}\left[\rightarrow_{2}\right]\left(Y_{i}\right)\right)$ by Propositions 1 and 2

Since $\alpha \circ \operatorname{pre}^{\tau^{*} a}\left[\rightarrow_{2}\right]\left(Y_{i}\right)$ is included into pre $\left[\rightarrow_{1}\right]\left(\left\{q_{i}\right\}\right)$ for all $i \in I$, we obtain that $\bigcup_{i \in I}\left(\alpha \circ \operatorname{pre}^{\tau^{*} a}\left[\rightarrow_{2}\right]\left(Y_{i}\right)\right)$ is included into $\bigcup_{i \in I}$ pre $\left[\rightarrow_{1}\right]\left(\left\{q_{i}\right\}\right)$. The latter is included into pre $\left[\rightarrow_{1}\right](X)$. 
Theorem 1. Let $\eta$ be the relation between the two transition systems $T S_{2}=$ $\left\langle S_{2}, A_{c t}, \rightarrow_{1}, l_{2}\right\rangle$ and $T S_{1}=\left\langle S_{1}, A_{c t}, \rightarrow_{1}, l_{1}\right\rangle$. Then $S_{2} \sqsubseteq_{\eta} S_{1}$.

We slightly adapt the proof from [16] to our case by using Lemma 1, Definition 2 and Definition 7 .

So, the modular refinement relation is an $\eta$-simulation. However, this result uses only Definition [5 of $\rho$. In the next section, we will point up the role played by the non-determinism.

\subsection{Modular Refinement as a Generalization of the Ready-Simulation}

In this section, we see the modular refinement relation $\eta$ as a generalization of the ready-simulation of [10].

For that we will keep the ready-set definition (see [9, 10]) for the abstract system as readies $\left(q_{1}\right)=\left\{a \in\right.$ Act $_{1}$ s.t. $\left.q_{1} \stackrel{a}{\rightarrow}_{1}\right\}$, and, we will simply replace $\rightarrow_{2}$ by $\Longrightarrow_{2}$ for the refined system, i.e., readies $\left(q_{2}\right)=\left\{a \in A c t_{1_{\tau}}\right.$ s.t. $\left.q_{2} \stackrel{a}{\Longrightarrow}{ }_{2}\right\}$. Moreover, we take into account the non-divergence of $\tau$.

Theorem 2. Let $T S_{1}=\left\langle S_{1}, A c t_{1}, \rightarrow_{1}, l_{1}\right\rangle$ and $T S_{2}=\left\langle S_{2}, A c t_{1 \tau}, \rightarrow_{2}, l_{2}\right\rangle$ be two transition systems. If $\left(q_{2} \eta q_{1}\right)$ then readies $\left(q_{1}\right)=\bigcup_{q_{2}} \bigcup_{\text {s.t. } q_{2} \eta q_{1}}$ readies $\left(q_{2}\right)$.

Proof. $\subseteq$ ) It is immediate by Definition [6]

$\supseteq$ ) It is immediate by Clauses 1 and 2 of Definition 5

Therefore, $\eta$ implies the equality of the readies as it is the case for the ready simulation of [10]. This result points up that the set of a module exiting actions is equal to the set of this module name (see Definition 4) exiting actions.

Furthermore, the equality of the readies allows us to define $\xi$ as follows:

Definition 9. Let $T S_{1}=\left\langle S_{1}\right.$, Act $\left._{1}, \rightarrow_{1}, l_{1}\right\rangle$ and $T S_{2}=\left\langle S_{2}\right.$, Act $\left._{1 \tau}, \rightarrow_{2}, l_{2}\right\rangle$ be respectively a transition system and its refinement. Let a be in Act $_{1}$. The relation $\xi \subseteq S_{1} \times S_{2}$ is the greatest binary relation included into $\rho^{-1}$ and satisfying the following clause:

(readies) $\left.\left(q_{1} \stackrel{a}{\rightarrow}{ }_{1} q_{1}^{\prime} \wedge q_{1} \xi q_{2}\right)\right) \Rightarrow\left(\right.$ readies $\left.\left(q_{1}\right)=\underset{q_{2} \text { s.t. } q_{1} \xi q_{2}}{\bigcup} \operatorname{readies}\left(q_{2}\right)\right)$

Theorem 3. Definition $[$ and Definition 9 are equivalent.

Proof. $\Rightarrow$ ) It is immediate by Theorem 2

$\Leftarrow)$ It is immediate because Clause readies of Definition 9 implies Clause nondeterminism of Definition 6 .

Notice that Definition 9 is easily implementable since it is enough to verify the equality of the readies when verifying that Definition 5 holds. 


\section{Compositionality of the Modular Refinement}

We give a result concerning the compositionality of the modular refinement relation $\eta$ w.r.t. a parallel composition operation which is important for the application of the modular refinement in practice since it allows to build complex systems by a parallel composition of components.

Definition 10. (Parallel composition) Let $T S_{i}=\left\langle S_{i}\right.$, Act $\left._{i}, \rightarrow_{i}, l_{i}\right\rangle$ and $T S_{j}=$ $\left\langle S_{j}, A_{c} t_{j}, \rightarrow_{j}, l_{j}\right\rangle$ be two transition systems. The parallel composition of $T S_{i}$ and $T S_{j}$ is $T S_{i} \| T S_{j} \stackrel{\text { def }}{=}\left\langle S, A c t_{\tau}, \rightarrow, l\right\rangle$ where $S$ is the set of the $q \| q^{\prime \prime}$ ( $q \in S_{i}$ and $\left.q^{\prime \prime} \in S_{j}\right)$, Act is the union of $A c t_{i}$ and $A c t_{j}, l$ is the product of $l_{i}$ and $l_{j}$. Let $\alpha \in$ Act $_{\tau}$, for $k \in\{i, j\}$, the transition relation $\rightarrow$ is defined by combining individual actions in parallel as follows.

$$
\text { [PAR1] } \frac{q \stackrel{\alpha}{\rightarrow}_{k} q^{\prime}}{q\left\|q^{\prime \prime} \stackrel{\alpha}{\rightarrow} q^{\prime}\right\| q^{\prime \prime}} \quad \text { [PAR2] } \frac{q \stackrel{\alpha}{\rightarrow}_{k} q^{\prime}}{q^{\prime \prime}\left\|q \stackrel{\alpha}{\rightarrow} q^{\prime \prime}\right\| q^{\prime}}
$$

This definition means that all moves of parallel composition are moves of either $T S_{1}$ or of $T S_{2}$.

In the following theorem, $\eta$ denotes the modular refinement relation linking transition systems as well as their parallel compositions.

Theorem 4. Let $q_{2} \eta q_{1}$ and $q_{4} \eta q_{3}$. We have:

1. $q_{2}\left\|q_{4} \eta q_{1}\right\| q_{3}$

2. $q_{4}\left\|q_{2} \eta q_{3}\right\| q_{1}$

Proof. We prove the result for 1 , the second proof being similar.

For the result 1 , we show that $\mathcal{S}$ verifies clauses of Definitions [5] and 6 , where

$$
\mathcal{S} \stackrel{\text { def }}{=}\left\{\left(q_{1}\left\|q_{3}, q_{2}\right\| q_{4}\right) \text { s.t. } q_{2} \eta q_{1} \& q_{4} \eta q_{3}\right\}
$$

for $q_{1}, q_{2}, q_{3}, q_{4} \in S$. Now, suppose $\left(q_{1}\left\|q_{3}, q_{2}\right\| q_{4}\right) \in \mathcal{S}$.

1. First, we consider Definition 5. Let $q_{2} \| q_{4} \stackrel{\alpha}{\rightarrow} \tilde{q}$ with $\alpha \in$ Act $_{\tau}$. There are four cases:

(a) $q_{2} \stackrel{a}{\rightarrow} 2 q_{2}^{\prime}$, and $\tilde{q}=q_{2}^{\prime} \| q_{4}$. Then, because $q_{2} \eta q_{1}$, we have $q_{1} \stackrel{a}{\rightarrow}{ }_{1} q_{1}^{\prime}$ with $q_{2}^{\prime} \eta q_{1}^{\prime}$ by Clause 1 of Definition 5 hence also $q_{1}\left\|q_{3} \stackrel{a}{\rightarrow} q_{1}^{\prime}\right\| q_{3}$ by [PAR1] rule of Definition [10, and $\left(q_{1}^{\prime}\left\|q_{3}, q_{2}^{\prime}\right\| q_{4}\right) \in \mathcal{S}$.

(b) $q_{4} \stackrel{a}{\rightarrow}_{4} q_{4}^{\prime}$, and $\tilde{q}=q_{2} \| q_{4}^{\prime}$. We get the result in the same way than the case above by using $[P A R 2]$ rule of Definition 10 .

(c) $\alpha=\tau$, and $q_{2} \stackrel{\tau}{\rightarrow} 2 q_{2}^{\prime}$. Moreover, we derive $q_{2}^{\prime} \stackrel{a}{\Longrightarrow} q_{2} q_{2}^{\prime \prime}$ and $q_{2} \| q_{4} \stackrel{\tau}{\rightarrow}$ $q_{2}^{\prime}\left\|q_{4} \stackrel{a}{\Longrightarrow} q_{2}^{\prime \prime}\right\| q_{4}(=\tilde{q})$. Then, because $q_{2} \eta q_{1}$, we have $q_{1} \stackrel{a}{\rightarrow} 1 q_{1}^{\prime}$ with $q_{2}^{\prime} \quad \eta q_{1}$ and $q_{2}^{\prime \prime} \eta q_{1}^{\prime}$ by Clause 2 of Definition [5. hence, we also get $q_{1}\left\|q_{3} \stackrel{a}{\rightarrow} q_{1}^{\prime}\right\| q_{3}$ by $[P A R 1]$ rule of Definition [10, and $\left(q_{1}\left\|q_{3}, q_{2}^{\prime}\right\| q_{4}\right)$ and $\left(q_{1}^{\prime}\left\|q_{3}, q_{2}^{\prime \prime}\right\| q_{4}\right)$ are in $\mathcal{S}$. 
(d) $\alpha=\tau$, and $q_{4} \stackrel{\tau}{\rightarrow}_{4} q_{4}^{\prime}$. We have the result by the same argument than the case above.

2. Second, we consider Definition 6] Let $q_{1} \| q_{3} \stackrel{\alpha}{\rightarrow} \tilde{q}$ with $\alpha \in$ Act. There are two cases:

(a) $q_{1} \stackrel{a}{\rightarrow} 1 q_{1}^{\prime}$, and $\tilde{q}=q_{1}^{\prime} \| q_{3}$. Then, because $q_{2} \eta q_{1}$, we have $q_{1} \xi q_{2}$. By Definition 6, there exists $q_{2}^{\prime}, q_{2}^{\prime \prime}$ s.t. $q_{1} \xi q_{2}^{\prime} \wedge q_{2}^{\prime} \stackrel{a}{\Longrightarrow} q_{2} q_{2}^{\prime \prime} \wedge q_{1}^{\prime} \xi q_{2}^{\prime \prime}$. By [PAR1] rule of Definition[10, we have $q_{2}^{\prime}\left\|q_{4}, q_{2}^{\prime \prime}\right\| q_{4}$ s.t. $q_{1}\left\|q_{3} \xi q_{2}^{\prime}\right\| q_{4} \wedge$ $\left.q_{2}^{\prime}\left\|q_{4} \stackrel{a}{\Longrightarrow} q_{2}^{\prime \prime}\right\| q_{4} \wedge q_{1}^{\prime}\left\|q_{3} \xi q_{2}^{\prime \prime}\right\| q_{4}\right)$, and $\left(q_{1}\left\|q_{3}, q_{2}^{\prime}\right\| q_{4}\right)$ and $\left(q_{1}^{\prime}\left\|q_{3}, q_{2}^{\prime \prime}\right\| q_{4}\right)$ are in $\mathcal{S}$.

(b) We have the same proof for the case $q_{3} \stackrel{a}{\rightarrow}_{3} q_{3}^{\prime}$ and $\tilde{q}=q_{1} \| q_{3}^{\prime}$.

This compositionality result permits the modular design of the system into separate components. So, the attribute "modular" of the refinement relation $\eta$ expresses the modular design ability as well as the partition of the refined system state space into modules.

\section{$7 \quad$ Example}

The definition of the modular refinement relation is motivated by the conditions expressed in terms of path set refinement as we saw in Section 4. We illustrate the refinement design with a simple example of a robot carrying parts inspired from [3. It shows how the modules appear in the refined reachability graph, and how the non-determinism decreases during the refinement process. This last point justifies the clause non-determinism of Definition 6. The robot's example is voluntarily simple. We have studied the modular refinement relation about less trivial applications such as the protocol $T=1$ [13], a BRP protocol [5] and so on.

We suppose the carrier device $(C D)$ carries one part at a time from an arrival device $A D$ located to its left towards two exit devices located respectively to the left $(E D L)$ and the right $(E D R)$ of the carrier device (see Figure 7).

The two refinement levels of transition systems (presented in Figure 8 and Figure [6 by their reachability graphs) model the transportation of parts by the carriage device on the exit devices. The first level ignores the movement of the carriage device and how parts arrive in it. The second level introduces the rotation movement of the carrier device.

At the first level of abstraction (see Figure 8), we are not concerned which of the exit devices is receiving the part. This introduces what we call an internal non-determinism in the abstract transition system. The variables are $C D, E D L$, $E D R$. Here, the invariant is well-typing: $C D, E D L, E D R \in\{$ free, busy $\}$. Initially, all the devices are free. The actions that can be observed are the loading of a part (label $L$ ), the deposit of a part (label $U$ ), the exit of a part from the left exit device (label $E x L$ ) and the exit of a part from the right exit device (label $E x R)$. The interpretation of the eight different states appears graphically. For example, $l\left(q_{5}\right)$ is $C D=$ busy $\wedge E D L=$ free $\wedge E D R=$ busy. One can notice two transitions labeled by $U$ originating from $q_{1}$ (internal non-determinism). 
The second level of refinement (see Figure [6) introduces the observation of the two rotations, from the left to the right $(R o R)$ and from the right to the left $(R o L)$ that the carrier device must do in order to deposit a part either to the left or to the right. The glue invariant is $I_{2} \stackrel{\text { def }}{=}\left(C D^{\prime}=C D \wedge E D L^{\prime}=\right.$ $\left.E D L \wedge E D R^{\prime}=E D R \wedge \operatorname{Pos} \in\{l, r\}\right)$.

A plausible behavior may remove the internal non-determinism by forcing the carrier device to unload on the left exit device if it is turned toward the left; respectively, to unload on the right exit device if it is turned toward the right (in position). Moreover, the rotation towards the left becomes a priority in the other situations since the arrival device is located to the left. This imposed behavior is supposed to minimize the number of rotations towards the right.

We verify that $S_{2}$ is a modular refinement of $S_{1}$ by substituting $\tau$ transitions by transitions labeled by $R o L, R o R$ and verifying Definitions 5] and 6] Moreover, one can notice how the refinement introduces a partition of the refined transition system into modules. Notice in the example that the two transitions labeled by $U$ exiting from the module of name $q_{1}$ are the reflection of the two transitions labeled by $U$ exiting from $q_{1}$ at the abstract level. So, the traces containing $U$ and beginning with $q_{1}$ remain. So, we have readies $\left(q_{1}\right)=\operatorname{readies}\left(q_{11}^{\prime}\right) \cup \operatorname{readies}\left(q_{12}^{\prime}\right)$ where $q_{11}^{\prime}$ and $q_{12}^{\prime}$ are the two states in the module of name $q_{1}$.

Another plausible behavior may remove the internal non-determinism by simply forcing the carrier device to unload on the left (left first) when both devices are free. This imposed behavior is, as above, supposed to optimize the number of rotations since the arrival device is located to the left.

Notice that there is only one transition labeled by $U$ exiting from the module $q_{1}$ which is the reflection of the two transitions labeled by $U$ exiting from $q_{1}$ at the abstract level. So, some traces containing $U$ and beginning by $q_{1}$ disappear between the abstract and the refined level but at least one trace containing $U$ remains. We can use other strategies such as, for example, forcing the carrier device to unload on the exit device which has been freed the first (first free, first busy).

\section{Application}

As safety and liveness properties are essential for reactive systems, the verification uses different methods which are based either on the model or on the system description. The model-based verification methods use the labeled transition systems techniques equipped with logics or behavior equivalences. The methods which are working on the system description via its denotational semantics, prove theorems about the system behavior by using an appropriate logic. The theorem provers or the proof assistants are semi-automatic in contrast with the model-checkers, but they can handle infinite systems.

To get the advantages of both methods in verifying that the B event system satisfies its dynamic properties (expressed by $L T L$ formulae), it is very interesting to be able to combine model-checking with theorem proving in the same verification tool set [8]. In [12, 14, 18, we propose an original combination of 
the above techniques. Proof is used when fully automatic (for example, tautology checker in propositional calculus). At the abstract level of the refinement, model-checking is used for dynamic property verification. The proposed refinement relation allows us to cross both techniques at the best of their advantages and possibilities. This is made possible because the proposed refinement relation determines modules in the refined reachability graph (for that, see []).

For finite state transition systems, the refinement verification and the module construction are implementable by an on-the-fly traversal of the refined transition system reachability graph. This is implemented as a component of a verification tool set [8].

\section{Conclusion and Related Works}

We are following an approach using both automatic-proof and model-checking technologies. This cooperation is permitted because of the refinement methodology [12, 14] which has been proposed to specify reactive systems and to verify their dynamic properties expressed in the $L T L$.

We have defined a formal framework for this methodology: the modular refinement relation between an abstract and a refined transition systems derived from the B design.

Since we apply the modular relation $\eta$ to finitely branching transition systems, and since $\eta$ does not accept the $\tau$-divergence, it is closely related to the refusal simulation as defined by Ulidowsky in 24. The refusal simulation is also a generalization of the ready simulation of Bloom et al. [10]. The main difference between the modular refinement relation $\eta$ and the refusal simulation is in the non-determinism clause of Definition 6. This clause requires that $q_{1}$ relates to $q_{2}$, i.e., that the module of name $q_{1}$ exists but the refusal simulation does not. This constraint is the key to obtain the equality of the readies (see Theorem 21). Therefore, the modular refinement relation, which is also a generalization of the ready simulation, seems to be strictly included in the refusal simulation.

We can also relate the clauses of Definition 5 to the clauses $(a),(\epsilon),(O),(\Delta)$, $(S)$ of van Glabbeek in [1] by taking into account of the fact that there is no silent moves in the abstract transition system. Therefore, we could situate the modular refinement relation as a divergence sensitive stability respecting completed simulation in the van Glabbeek' spectrum.

Another contribution of this work is that it gives a good semantics which authorizes us to compare the modular refinement relation with other refinement definitions [20, 15]. We anticipate that without the non-determinism, it is also equivalent to Lamport's TLA refinement.

One of the consequences of Theorem 4 is that the modular refinement relation is preserved w.r.t. the parallel composition. This allows us to keep the same refinement notion for both modular design and modular verification of reactive systems.

The partition of the refined system state space into modules is the second reason to call modular our refinement relation. It facilitates the verification by 
taking into account the system modularity introduced by the refinement. Accordingly we are currently building the verification tool set 8 .

\section{References}

[1] J. R. Abrial. The B Book. Cambridge University Press - ISBN 0521-496195, 1996.

[2] J. R. Abrial. Extending B without changing it (for developing distributed systems). In 1st Conference on the B method, pages 169-190, Nantes, France, November 1996 .

[3] J. R. Abrial. Constructions d'automatismes industriels avec B. In Congrès AFADL, ONERA-CERT - Toulouse, France, May 1997. Invited lecture.

[4] J. R. Abrial, E. Börger, and H. Langmoeck. Specifying and Programming the Steam Boiler Control. LNCS 1165. Springer Verlag, 1996.

[5] J. R. Abrial and L. Mussat. Specification and design of a transmission protocol by successive refinements using B. LNCS, 1997.

[6] J. R. Abrial and L. Mussat. Introducing dynamic constraints in B. In Second Conference on the B method, LNCS 1393, pages 83-128, Montpellier, France, April 1998. Springer Verlag.

[7] P. Behm, P. Desforges, and J.M. Meynadier. MÉTÉOR: An industrial success in formal development. In Second conference on the B method, LNCS 1393, Montpellier, France, April 1998. Springer Verlag. Invited lecture.

[8] F. Bellegarde, J. Julliand, and H. Mountassir. Model-based verification through refinement of finite B event systems. In Formal Method'99 B User Group Meeting, CD-ROM publication, 1999.

[9] B. Bloom. Ready Simulation, Bisimulation, and the Semantics of CCS-Like Languages. PhD thesis, MIT, August 1989.

[10] B. Bloom, S. Istrail, and A. R. Meyer. Bisimulation can't be traced. Journal of the ACM, 42(1):232-268, January 1995.

[11] R. J. van Glabbeek. The linear time - branching time spectrum II: The semantics of sequential systems with silent moves. In Proc. CONCUR'93, Hildesheim, Germany, LNCS 715, pages 66-81. Springer-Verlag, August 1993.

[12] J. Julliand, F. Bellegarde, and B. Parreaux. De l'expression des besoins à l'expression formelle des propriétés dynamiques. Technique et Science Informatiques, 18(7), 1999.

[13] J. Julliand, B. Legeard, T. Machicoane, B. Parreaux, and B. Tatibouet. Specification of an integrated circuits card protocol application using B and linear temporal logic. In Second conference on the B method, LNCS 1393, pages 273292, Montpellier, France, April 1998. Springer Verlag.

[14] J. Julliand, P.A. Masson, and H. Mountassir. Modular verification of dynamic properties for reactive systems. In International Workshop on Integrated Formal Methods (IFM'99), York, Great Britain, 1999.

[15] L. Lamport. A temporal logic of actions. 16:872-923, May 1994.

[16] C. Loiseaux, S. Graf, J. Sifakis, A. Bouajjani, and S. Bensalem. Property preserving abstractions for the verification of concurrent systems. Formal Methods in System Design, 6:1-35, January 1995.

[17] R. Milner. Communication and Concurrency. Prentice Hall Int., 1989.

[18] H. Mountassir, F. Bellegarde, J. Julliand, and P.A. Masson. Coopération entre preuve et model-checking pour vérifier des propriétés LTL. In submission AFADL'2000, 2000. 
[19] O. Ore. Galois connections. Trans. Amer. Math. Soc., (55):493-513, February 1944.

[20] A. Pnueli. System specification and refinement in temporal logic. In Proc. 12th Conf. Found. of Software Technology and Theor. Comp. Sci., New Delhi, India, LNCS 652, pages 1-38. Springer-Verlag, December 1992.

[21] L. E. Sanchis. Data types as lattices : retractions, closures and projections. RAIRO Informatique Théorique et Applications, 11(4):329-344, 1977.

[22] J. Sifakis. Property preserving homomorphisms of transition systems. In Proc. Logics of Programs Workshop, Pittsburgh, LNCS 164, pages 458-473. SpringerVerlag, June 1983.

[23] H. Treharne, J. Draper, and S. Schneider. Test case preparation using a prototype. In Second conference on the B method, LNCS 1393, pages 293-312, Montpellier, France, April 1998. Springer Verlag.

[24] I. Ulidowski. Equivalences on observable processes. In Proceedings of the 7th Annual IEEE Symposium on Logic in Computer Sciences IEEE, New-York, IEEE Computer Society Press, pages 148-161, 1992.
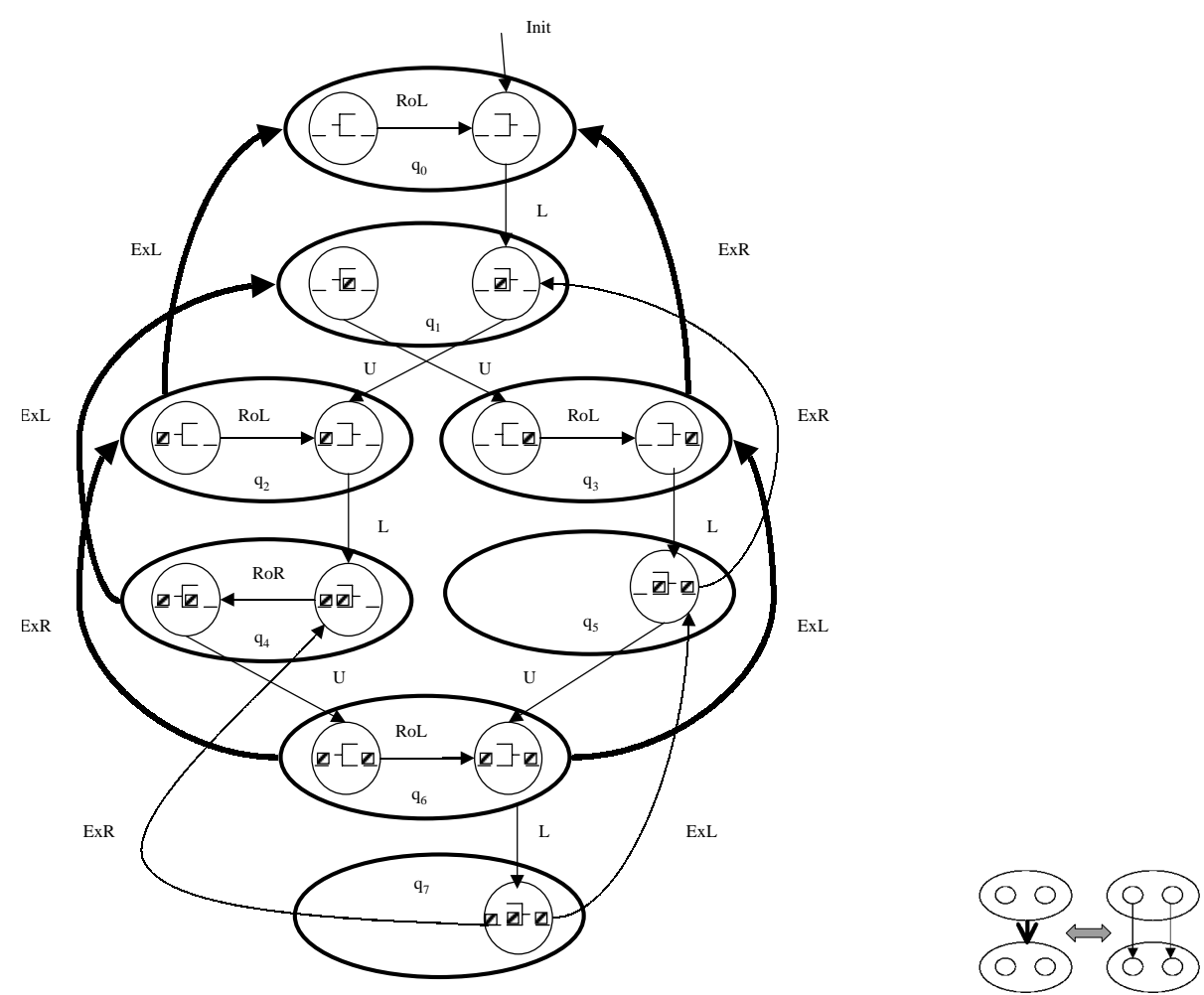

Fig. 6. A refined transition system (in position). 


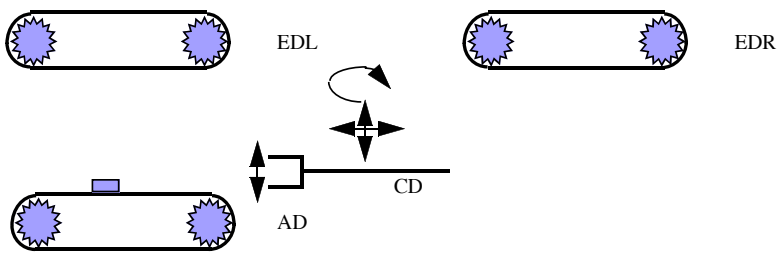

Fig. 7. The physical system.

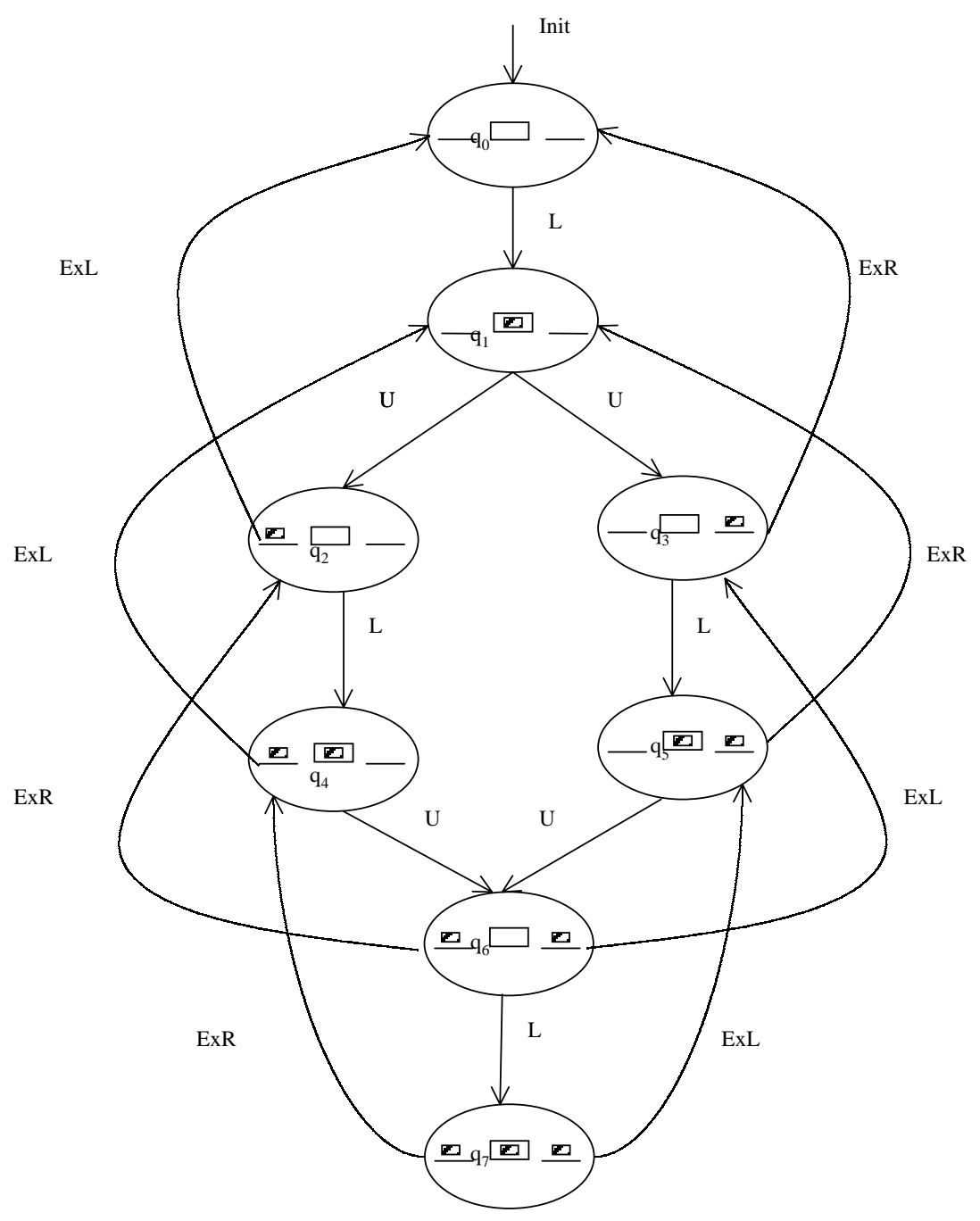

Fig. 8. The abstract transition system. 